Paper published as:

Tancredi, U. \& Grassi, M. (2007), 'Approximate trajectories for thermal protection system flight tests mission design', Journal of Spacecraft and Rockets 44(5), 1003--1011.

http://arc.aiaa.org/doi/abs/10.2514/1.22007

Copyright 2007 by the American Institute of Aeronautics and Astronautics, Inc. 


\title{
Approximate trajectories for Thermal Protection System flight tests mission design
}

\author{
Urbano Tancredi ${ }^{1}$ and Michele Grassi ${ }^{2}$ \\ University of Naples Federico II, Napoli, 80125, Italy
}

\begin{abstract}
A mission profile for advanced Thermal Protection System suborbital flight testing is identified. Its main goal is to achieve a constant heat flux at a specific area of the vehicle for a limited amount of time. A tool capable of exploring broad regions of the design space for these missions is developed, aiming at reducing possible design options to an extent manageable by conventional, more accurate, numeric-simulation-based methods. Based on a simplified model of the point mass dynamics, trajectories optimal for Thermal Protection System testing and compliant with prefixed path constraints are identified. The approximate method is validated comparing the obtained optimal trajectories with numeric-optimized standard solutions on three test cases. Then, to demonstrate the method effectiveness and flexibility, the mission design space is investigated for reasonable ranges of relevant parameters. Results show that increasing the vehicle's ballistic coefficient allows reducing the specific mechanical energy at reentry, and that the maximum admissible dynamic pressure plays a principal role in affecting the attainable testing performances. An illustrative mission design for novel ceramic Thermal Protection System testing is presented, that minimizes in the analyzed design space the specific mechanical energy at the trajectory apogee.
\end{abstract}

\footnotetext{
${ }^{1}$ Ph.D. in Aerospace Engineering, Department of Aerospace Engineering, P.le V. Tecchio 80, AIAA Student Member

${ }^{2}$ Associate Professor, Department of Aerospace Engineering, P.le V. Tecchio 80.
} 


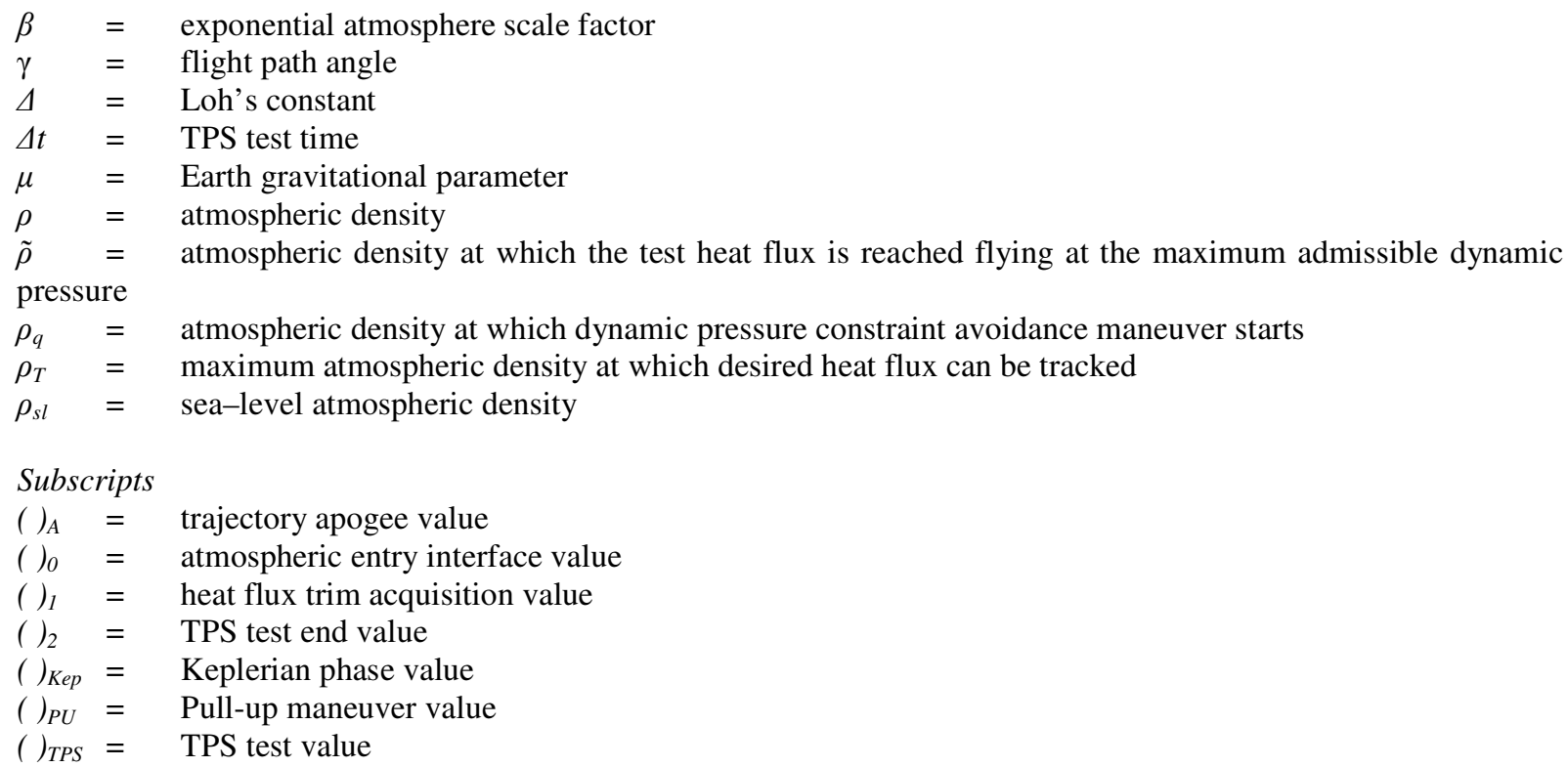

\section{Introduction}

THE Thermal Protection System ( TPS ) is one of the most critical subsystems in vehicles performing an atmospheric reentry. Hence, a high confidence level must be acquired prior to integration of this subsystem into a flight vehicle. This requires intensive ground and flight testing activities to validate novel TPS concepts. TPS ground testing poses several non trivial challenges, mainly related to the reproduction of the aero-thermal flight conditions. Purposely conceived ground facilities are required to replicate the challenging aero-thermal environment. Usually the resulting plants ${ }^{1}$ are huge, complex, and remarkably costly to develop and operate. In face of these difficulties, the capability to reproduce on ground the reentry flight aero-thermal environment is still significantly limited ${ }^{2}$. As a consequence, the flight demonstration of a ground validated TPS has some degree of associated risk. Specifically designed flight tests are thus a viable alternative.

To acquire thorough confidence in the TPS performance, the most effective flight test strategy is to perform a reentry from an orbital (or near orbital) initial state, flying in aero-thermal conditions close to the ones in which the TPS is intended to operate. On the other hand, such a testing approach requires to perform an entire atmospheric reentry, with resulting high cost and complexity. Nonetheless, these must be kept low when dealing with a subsystem flight test, or a high risk technology demonstration, as in the case of novel TPS validation. Indeed, recent examples of this strategy either incorporate testing of several reentry subsystems, as in the case of the Japanese OREX (Orbital Re-entry Experiment) flight ${ }^{3}$, or are designed for thorough characterization of the reentry aero- 
thermal environment by means of multiple experiments, as for the upcoming European EXPERT (European Experimental Re-entry Testbed) mission ${ }^{4}$.

Thus, atmospheric reentry from orbital or high-energy suborbital conditions does not seem a viable option for flight testing of advanced TPS by means of a purposely designed mission. Hence, we do not cover these cases in the present paper. Significant savings in the resources needed to perform a TPS flight test can be obtained if the flight reaches only low-energy suborbital conditions, possibly making maximum use of off-the-shelf vectors, such as ballistic missiles or sounding rockets ${ }^{5,6}$. The main drawback of this flight testing strategy is that TPS testing is carried out in a similar, but different, aero-thermal environment. For instance, the heat flux time profile differs from reentry ones, even if the values can be comparable. The trajectory covers different Reynolds number and dynamic pressure regions, which influence the onset of complex phenomena, such as boundary layer transition. It is clear that this difference between the flight test conditions and the operating ones shall be taken into account for test results' interpretation. This is thought to be an important issue in such a testing strategy. Nonetheless, this subject is beyond the scope of the present paper, that is intended to focus on simplified models for mission analysis and design of such flight tests. Recent examples of suborbital TPS flight tests include the German SHEFEX ( SHarp Edge Flight EXperiment) mission ${ }^{6}$, whose main focus is on hypersonic aero-thermodynamic data gathering for faceted surfaces and sharp edges, NASA's SHARP - B ( Slender Hypersonic Aerothermodynamic Research Program ) ${ }^{7}$, and the SRT ( Suborbital Reentry Test ) of the Unmanned Space Vehicle program ${ }^{8}$, currently under development at the Italian Aerospace Research Center ( CIRA ).

All these missions experience an extremely variable heat flux time profile, typical of low aerodynamic efficiency orbital reentry flights, although heat flux values are lower due to the sensibily smaller specific mechanical energy. Conversely, in a lifting reentry the heat flux tends to be more constant during the maximum heat flux flight portion ${ }^{9}$. In the present work we will focus on a testing approach that is somehow in between a complete atmospheric reentry and the suborbital ballistic flights just mentioned. Specifically, the flight test starts as well from low suborbital energy levels, but is designed to obtain constant heat flux conditions on a TPS sample. This approach allows a better characterization of the TPS behavior versus input heat fluxes in conditions more similar to lifting reentry, and simplifies the test results interpretation, that benefits from the constant thermal input.

Suborbital TPS flight tests constitute a peculiar application from the point of view of trajectory determination. In order to replicate the heat flux values typical of atmospheric reentry, the trajectory shall be designed to maximize the 
aero-thermal stress on the vehicle, exactly the opposite of what is customary done in reentry trajectory design. More precisely, atmospheric reentry trajectories are usually determined constraining, among others, the heat flux of some vehicle's specific point(s) to be lower than a given threshold, dictated by the selected TPS materials. Then, the trajectory is obtained minimizing the integrated heat load on the vehicle, which mainly determines the TPS mass. For suborbital flight tests, given the lower energy levels, the integrated heat load is not the primary concern. In order to obtain the lowest launch energy that realizes the desired aero-thermal environment, the aero-thermal stress experienced by the vehicle shall thus be maximized.

Given the peculiarity of these applications, there is the need of developing appropriate mission analysis tools, e.g. capable of determining, for desired TPS testing flight conditions, the specific mechanical energy levels and the vehicle's aerodynamic characteristics needed to satisfactorily perform the flight test. Previous studies related to similar missions have been focused on the specific system and mission needs, by applying and/or adapting conventional mission and trajectory design techniques typical of reentry problems ${ }^{10,11}$. A parametric analysis is performed in the mission design space, making use of local numeric optimization schemes for trajectory design, carried out via massive numeric dynamics simulations ${ }^{10-13}$. The main limitation of these techniques is the capability of exploring only a limited number of candidate solutions in the mission design space, that are usually identified based on the legacy of similar missions. In applications that do not benefit of previous experiences, such as those we refer to, the design space to be evaluated by parametric analysis is often restricted by means of heuristic interpretation of the underlying physics. In this context, a tool capable of exploring broad regions of the mission design space would be extremely helpful in narrowing the possible design candidates to an extent manageable by numeric-simulation-based methods. To the authors knowledge, no such tools have ever been developed to aid in investigating optimal mission designs for constant heat flux suborbital TPS flight tests.

The objective of the present paper is to derive a method capable to perform mission analysis over a wide portion of the design space of such missions, by means of simplified analytical dynamics models suitable to identify optimal trajectories. The tool is intendended for a mission design phase, being more flexible than numeric-simulation-based methods in analyzing broad regions of the parameter's design space. The purpose of the method is to assess the main features of a mission design capable to meet the desired performances, in order to determine design candidates for further analysis. Therefore, it should be considered as preparatory and complementary to more accurate numericsimulation-based methods. In the following, a class of trajectories is identified that responds to the TPS testing 
requirements, on which dynamics modeling issues are analyzed. A simplified, semi-analytic model of the point mass dynamics in the longitudinal flight plane is proposed. Based on this model, trajectories optimal for TPS testing and compliant with given path constraints are identified. Then, the accuracy of the simplified approach is evaluated by comparison with numeric-optimized standard solutions on three test cases. Finally, to exemplify the method effectiveness and flexibility, the mission design space is investigated for reasonable ranges of relevant parameters, yielding to trajectory initial conditions that allow performing the TPS flight test as desired.

\section{Simplified Dynamics Models}

As usually done in mission design phases, a point mass dynamics model is used to describe the vehicle's trajectory. In addition, only the motion in the longitudinal plane is considered, since no requirements are imposed on the trajectory cross-range. The resulting dynamics is described by a two degree of freedom ( DOF ) model, whose representation can be given as in Eq.(1). We emphasize that L/D stands for the ratio between lift vertical component and drag, whereas the vehicle's aerodynamic efficiency is denoted by $\mathrm{L}_{\mathrm{T}} / \mathrm{D}$.

$$
\dot{x}=f(x, u) \quad x=(V, \gamma, \rho)^{T} \quad u=(L / D, B)^{T}
$$

The point mass motion is described as planar entry flight over a spherical, nonrotating planet with constant gravity acceleration, exponential atmospheric density and zero thrust ${ }^{14}$. Concerning aero-thermal modeling issues, in atmospheric entry mission analysis and trajectory design, heat flux requirements are usually expressed in terms of the heat flux at one (or more) stagnation point ${ }^{15-17}$. Of course, since the TPS sample to be tested extends beyond the stagnation point, the incoming heat flux distribution over the testing area shall be determined as well. Computation of this distribution shall take into account complex mutual dependencies among vehicle geometry, surface characteristics, and flight conditions ${ }^{18}$. Heavily nonlinear phenomena can also occur, such as boundary layer transition, whose accurate prediction is still an active research topic ${ }^{19}$. Taking into account the incoming heat flux distribution thus requires a detailed knowledge of the system features. This prevents dealing with this subject in a mission design context. On the other hand, formulating TPS test requirements in terms of the stagnation point heat flux assures that the desired thermal input is obtained at the stagnation point. In addition, only system-level information is required to compute its value. Hence, we enforce TPS requirements referring only to the stagnation point heat flux, determined by means of the well-known Fay - Riddel formulation ${ }^{20}$. 


$$
\dot{q}=C \sqrt{\rho / R_{N}} \cdot V^{3}
$$

The $u$ vector in Eq.(1) characterizes maneuvering capability of the vehicle. We will assume that the aerodynamic shape of the vehicle is given, whereas its surface to mass ratio is a design parameter we wish to determine. Moreover, we will suppose the trajectory is flown at constant angle of attack. The angle of attack substantially affects the aero-thermal field surrounding the vehicle and the incoming heat flux distribution on its surface. For advanced TPS testing is highly desirable to have the test heat flux confined to a specific area of the vehicle, where the TPS sample is placed. This constrains the angle of attack to be somewhat near a certain value, ruling out angle of attack guidance. As a consequence, the ballistic coefficient can be assumed to be constant along the trajectory. Its value is determined by the vehicle's mass to surface ratio and the drag coefficient arising from the selected angle of attack. We thus assume that the vehicle maneuvers by rolling the lift vector about the velocity vector, a control technique known as bank modulation. In this way the vehicle can fly anywhere between a full lift up $\left(0^{\circ}\right.$ bank $)$ and full lift down $\left(180^{\circ}\right.$ bank) orientation as required for trajectory tracking. As a consequence, the L/D on a generic point of the trajectory is between the negative and positive $\mathrm{L}_{\mathrm{T}} / \mathrm{D}$ corresponding to the chosen angle of attack. It is worth noting that, to benefit from higher maneuvering capability, the angle of attack should be selected to maximize the aerodynamic efficiency.

Since the trajectory is conceived to maximize the aero-thermal stress on the vehicle, the ascent phase ends outside the atmosphere, to minimize launch energy losses. A wealth of analytic methods is available for ascent dynamics description ${ }^{14}$. We thus restrict the analysis to the reentry phase of the flight. In other words, we assume that the mission starts at the trajectory "apogee", where the altitude is maximum and the flight path angle is zero.

In order to obtain the equations of motion in closed form, the mission profile is splitted into three main phases. This is achieved by dividing the trajectory into three segments: a Keplerian phase, lasting until aerodynamics forces become significant, a pull-up maneuver, executed to achieve constant- $\dot{q}$ conditions for TPS testing, and a TPS test phase, performed at constant heat flux. As a result, we obtain three different simplified dynamics models, that patched together yield the complete equations of motion. An interpretation is given in Fig.1, where a typical trajectory is shown in the density, velocity plane. 


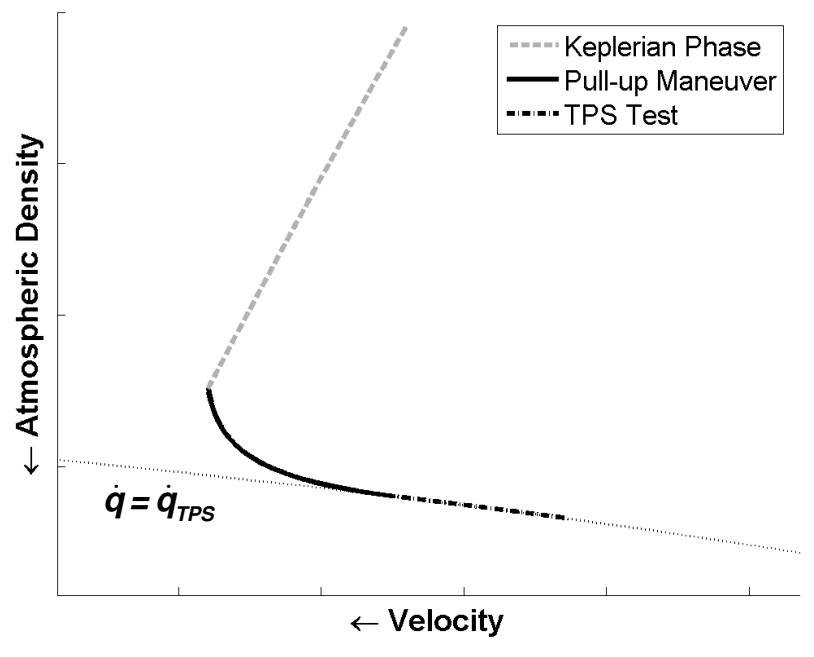

Fig. 1 Typical TPS flight test reentry trajectory

For the sake of clarity, in the following sections the three models are introduced starting from the last trajectory segment.

\section{A. TPS Test Phase}

We assume that the TPS test is carried out on a trajectory with small flight path angles. As it will be clearer later on, this is because aero-thermal stress maximization requires a trajectory as horizontal as possible. The gravity tangential component can thus be neglected. The resulting equations of motion can be written in local form as

$$
\begin{gathered}
d V / d \rho=V / 2 \beta \gamma B \\
d(\cos \gamma) / d \rho=\frac{L / D}{2 \beta B}-\frac{\left(g R / V^{2}-1\right)}{\rho \beta R}
\end{gathered}
$$

Enforcing heat flux trim conditions by means of Eq.(2), the velocity and flight path angle become a function of the atmospheric density.

$$
\begin{gathered}
V_{T P S}(\rho)=k \rho^{-1 / 6} ; \gamma_{T P S}(\rho)=-3 \rho / \beta B \\
k:=\left(\dot{q}_{T P S} \sqrt{R_{N}} / C\right)^{1 / 3}
\end{gathered}
$$


From Eqs.(3),(6), we can arrive at an expression for the time spent flying between two points on a constant heat flux trajectory. Using the kinematic relationship between altitude and flight path angle, after some manipulations we get

$$
\Delta t=2 B\left(\rho_{1}^{-5 / 6}-\rho_{2}^{-5 / 6}\right) / 5 k
$$

Thus, aero-thermal stress maximization can be viewed either as maximizing the test heat flux, i.e. maximizing $\mathrm{k}$ for a given test time $\Delta \mathrm{t}$, or, conversely, maximizing $\Delta \mathrm{t}$ for a given $\mathrm{k}$. Eq. (7) implies that both these two alternative formulations require B maximization. This, in turn, requires a constant- $\dot{q}$ trajectory as horizontal as possible ( see Eq. (5)).

In order to track the constant- $\dot{q}$ trajectory, the vehicle shall have the necessary maneuvering capability. Tracking a constant- $\dot{q}$ trajectory requires a specific L/D at each atmospheric density, depending on the ballistic coefficient value. Indeed, substituting the trajectory Eq. (5) in Eq. (4) and rearranging in terms of the vertical lift-to-drag ratio yields

$$
L / D_{T P S}=\frac{2 B}{R \rho} \cdot\left(\frac{g R}{k^{2}} \rho^{1 / 3}-1\right)-\frac{18 \rho}{\beta B}
$$

As a matter of fact, in order to track the constant heat flux trajectory, the requested $\mathrm{L} / \mathrm{D}_{\mathrm{TPS}}$ shall be within \pm $\mathrm{L}_{\mathrm{T}} / \mathrm{D}$. To estimate the density range in which this condition is met, we assume $\mathrm{L} / \mathrm{D}_{\mathrm{TPS}}$ is a monotone decreasing function of the atmospheric density. Inspection of Eq. (8) shows that this holds as far as the centripetal acceleration due to Earth curvature has a secondary effect. While this is true for low-energy suborbital flight, this assumption shall be carefully applied when dealing with near-orbital specific mechanical energy levels. In fact, in closed orbits this term balances the gravitational acceleration effect. As a consequence of this simplification, the minimum and maximum densities between which the vehicle can track $\dot{q}_{T P S}$ are obtained by substituting in Eq. (8) positive and negative $\mathrm{L}_{\mathrm{T}} / \mathrm{D}$, respectively. For instance, the maximum trackable density $\rho_{\mathrm{T}}$ satisfies the following nonlinear scalar equations, that can be solved by means of a numeric root finding scheme.

$$
\frac{2 B}{R \rho_{T}} \cdot\left(\frac{g R}{k^{2}} \rho_{T}^{1 / 3}-1\right)-\frac{18 \rho_{T}}{\beta B}+L_{T} / D=0
$$

\section{B. Pull-up Maneuver}


The model used to describe this trajectory segment is known as Loh's model ${ }^{21}$, originally conceived for atmospheric reentry. It can be derived by the 2-DOF point mass model by assuming that gravity tangential component is negligible and the difference between centripetal and vertical accelerations due to gravity is constant along the maneuver.

$$
\Delta:=2\left(g R / V^{2}-1\right) \cos \gamma / \rho R \cong \text { const. }
$$

Loh's model additionally assumes constant aerodynamic inputs, thus arriving at the closed form Eqs.(11),(12). Thus, we refer to a pull-up maneuver performed with constant L/D, i.e. with a constant bank angle.

$$
\begin{gathered}
V_{P U}(\rho)=V_{0} \cdot \exp \left(\frac{\gamma_{0}-\gamma_{P U}(\rho)}{L / D_{P U}-\Delta \cdot B}\right) \\
\gamma_{P U}(\rho)=\cos ^{-1}\left[\cos \gamma_{0}+\frac{1}{2 \beta}\left(\frac{L / D_{P U}}{B}-\Delta\right) \cdot\left(\rho-\rho_{0}\right)\right]
\end{gathered}
$$

Since the pull-up manuever is executed to acquire heat flux trim conditions, it ends on the constant heat flux trajectory segment. Enforcing this patching condition in Eqs. (11), (12) and rearranging, we obtain the analytic expressions relating the pull-up initial conditions to the trim acquisition state by means of the applied trajectory inputs.

$$
\begin{gathered}
L / D_{P U}=\Delta \cdot B+\left(\gamma_{0}+3 \rho_{1} / \beta B\right) / \ln \left(k \rho_{1}^{-1 / 6} / V_{0}\right) \\
2 B \beta-\frac{\gamma_{0}+3 \rho_{1} / \beta B}{\cos \left(-3 \rho_{1} / \beta B\right)-\cos \gamma_{0}} \cdot \frac{\rho_{1}-\rho_{0}}{\ln \left(k \rho_{1}^{-1 / 6} / V_{0}\right)}=0
\end{gathered}
$$

\section{Keplerian Phase}

In this phase the point mass dynamics are assumed to be adequately described by the Keplerian model. Unlike the two preceding models, it uses the inverse-square gravity approximation. For our purposes, it is convenient to define the zero reference of gravity potential energy at zero altitude. Hence, given the apogee radius and the specific mechanical energy, we obtain the velocity and flight path angle as a function of either altitude, or atmospheric density via the exponential model. 


$$
\begin{gathered}
V_{K e p}(\rho)=\sqrt{2\left[E-\mu\left(R^{-1}-r(\rho)^{-1}\right)\right]} \\
\gamma_{K e p}(\rho)=\cos ^{-1}\left(\frac{r_{A}}{r(\rho)} \cdot \sqrt{\left[E-\mu\left(R^{-1}-r_{A}^{-1}\right)\right] /\left[E-\mu\left(R^{-1}-r(\rho)^{-1}\right)\right]}\right) \\
r(\rho)=R-\beta^{-1} \ln \left(\rho_{s l} / \rho\right)
\end{gathered}
$$

The Keplerian phase ends when the drag loads due to the aerodynamic pressure become significant with respect to gravity ${ }^{22}$. This condition is here expressed in terms of dynamic pressure, i.e. when the dynamic pressure exceeds a threshold value the Keplerian model ceases to be valid. In this way, the density at which the flight becomes atmospheric can be obtained by specifying an appropriate dynamic pressure threshold. This yields to the nonlinear Eq. (18), whose solution $\rho_{0}$, computed by a numeric root finding scheme, allows determining the patching conditions with the pull-up maneuver via Eqs. (15), (16).

$$
\rho_{0}\left[E-\mu\left(\frac{1}{R}-\frac{\beta}{\beta R-\ln \left(\rho_{s l} / \rho_{0}\right)}\right)\right]-\bar{q}_{A t m}=0
$$

\section{Approximate Model Formulation and Trajectory Determination}

In order to correctly estimate the performance achievable by a generic mission design, the trajectories on which the approximate method is based are determined solving an optimization problem, in which the merit function is the aero-thermal stress on the test material. As pointed out in section II.A, this is equivalent to maximize the TPS test time.

Path constraints are enforced on the maximum heat flux and the maximum dynamic pressure. Since we refer to advanced TPS testing applications, the desired test heat flux is also the maximum the TPS can sustain without damage. The heat flux constraint is thus implicitly satisfied requiring to fly in proximity of its test value. Furthermore, it can be shown the maximum dynamic pressure constraint specifies a maximum density above which the TPS test cannot take place.

A constant dynamic pressure specifies a curve in the V- $\rho$ plane. In order to comply with the constraint, the vehicle shall fly geometrically above that curve, i.e. at densities lower than the one on the maximum dynamic 
pressure curve for each velocity value. The constant heat flux specifies a curve in the V- $\rho$ plane as well, and there is a unique point in which the two curves intersect.

$$
\tilde{\rho}=\left(\sqrt{2 \bar{q}_{\text {Max }}} / k\right)^{3}
$$

For densities lower than $\tilde{\rho}$, the constant- $\dot{q}$ curve is geometrically above the constant- $\bar{q}$ one. Therefore, to comply with the maximum dynamic pressure constraint, the TPS test can be performed only at densities lower than $\tilde{\rho}$. Nevertheless, this condition does not guarantee by itself compliance with the dynamic pressure constraint. Indeed, a flight path angle discontinuity occurs at the intersection point between the $\dot{q}_{T P S}$ and the $\bar{q}_{\text {Max }}$ curves, that has to be taken into account. Proceeding as in section II.A, we can express the flight path angle required to fly on the $\bar{q}_{\text {Max }}$ curve as

$$
\left.\gamma\right|_{\bar{q}=\text { const. }}=-\rho / \beta B
$$

Comparing Eqs. (5), (20) it is seen that a constant heat flux curve requires a steeper flight path than a constant dynamic pressure one. To transfer from the $\dot{q}_{T P S}$ to the $\bar{q}_{M a x}$ curve the vehicle shall execute an additional pull-up maneuver. This maneuver shall start at a density adequately lower than $\tilde{\rho}$, so that it is possible to increase the flight path angle at least up to the $\bar{q}_{\text {Max }}$ curve one. The maximum dynamic pressure constraint effect is thus to specify a superior limit to the density range in which the TPS test can take place.

An estimate of the test end density is obtained introducing additional simplifying assumptions. Specifically, we refer to an "efficient" pull-up, i.e. that allows performing the TPS test up to the highest possible density. This implies the maneuver makes use of maximum vertical lift-to-drag ratio, and the resulting trajectory is tangent to the $\bar{q}_{M a x}$ constraint when the maneuver ends. In addition, since the aerodynamic forces attain a maximum value, gravity can be neglected. At last, we assume the pull-up ends where the $\dot{q}_{T P S}$ and $\bar{q}_{M a x}$ curves intersect. Although no feasible maneuver exists matching this assumption, it allows deriving a simple end density estimate. Hence, Eq. (11) can be modified to get

$$
\frac{1}{6} \ln \left(\frac{\rho_{2}}{\tilde{\rho}}\right)+\frac{3 \rho_{2}-\tilde{\rho}}{\beta B \cdot L_{T} / D}=0
$$

A simple $\rho_{2}$ estimate can be achieved noting that a solution to Eq. (21) exists if and only if $1 / 3<\rho_{2} / \tilde{\rho}<1$. The pull-up should start at $\tilde{\rho} / 3$ in case of zero $\mathrm{L}_{\mathrm{T}} / \mathrm{D} \cdot \mathrm{B}$, whereas infinite $\mathrm{L}_{\mathrm{T}} / \mathrm{D} \cdot \mathrm{B}$ would allow performing the maneuver 
instantaneously at $\tilde{\rho}$. In order to achieve a reasonable compromise between model accuracy and simplicity, the $\rho_{2} / \tilde{\rho}$ ratio is assumed to be constant and equal to $3 / 4$ ( Eq. (22) ). It is worth noting that this $\rho_{2}$ estimate can be either conservative or not, depending on $\mathrm{L}_{\mathrm{T}} / \mathrm{D} \cdot \mathrm{B}$ and $\tilde{\rho}$. However, for a given $\tilde{\rho}$, the estimate becomes more conservative as $\mathrm{L}_{\mathrm{T}} / \mathrm{D} \cdot \mathrm{B}$ increases.

$$
\rho_{q}=3 / 4 \cdot\left(\sqrt{2 \bar{q}_{M a x}} / k\right)^{3}
$$

The maximum density at which the TPS test can be performed is also affected by the vehicle's capability to track the constant- $\dot{q}_{T P S}$ trajectory, as it has been shown in section II.A. More precisely, the TPS test can be carried out only within a limited density range, dictated by the vehicle's ballistic coefficient and aerodynamic efficiency. The minimum trackable density is not of concern, since it will be surely smaller than the thermal trim acquisition one. On the contrary, the maximum trackable density can be evaluated solving Eq. (9) with a numeric root finding scheme. The maximum density at which the TPS test can be performed $\rho_{2}$ is thus given by the minimum between those specified by the $\bar{q}_{M a x}$ constraint and the $\dot{q}_{T P S}$ tracking requirement.

$$
\rho_{2}=\min \left\{\rho_{q}, \rho_{T}\right\}
$$

In order to obtain the optimal trajectory, the variable to be optimized are the trajectory inputs $\mathrm{L} / \mathrm{D}$ and $\mathrm{B}$. However, given the approximate point mass dynamics model introduced in the previous sections, the inputs are determined once the pull-up maneuver vertical lift-to-drag ratio is specified. Indeed, the ballistic coefficient is determined by $\mathrm{L} / \mathrm{D}_{\mathrm{PU}}$ from Eqs. (13), (14), and $\mathrm{L} / \mathrm{D}_{\mathrm{TPS}}$ is univocally determined by the ballistic coefficient via the heat flux tracking condition ( Eq. (8) ). As a consequence, the optimization problem can be formulated as searching for the pull-up maneuver vertical lift-to-drag ratio that maximizes the TPS testing time $\Delta \mathrm{t}$, under the dynamics constraint Eqs. (13) - (16), (18), path constraints, which effect has been modeled with Eq. (23), and for a given initial state at the apogee .

$$
\begin{aligned}
& \Delta t\left(L / D_{P U}^{O P t}\right)=\max _{L / D_{P U} \in\left[-L_{T} / D, L_{T} / D\right]} \Delta t\left(L / D_{P U}\right) \\
& \text { s.t. } \\
& \text { Eqs.(13)-(16),(18),(23) } \\
& x\left(t_{A}\right)=x_{A}
\end{aligned}
$$


Solving problem (24) is not a trivial task, since, in general, $\rho_{2}$ depends nonlinearly on $\mathrm{L} / \mathrm{D}_{\mathrm{PU}}$ via the ballistic coefficient B. Nevertheless, if the $\dot{q}_{T P S}$ tracking specifies a maximum TPS test density higher than the dynamic pressure constraint one, the $\mathrm{L} / \mathrm{D}_{\mathrm{PU}}$ does not affect $\rho_{2}$. As it will be shown in the last section of this paper, this condition occurs for the majority of the cases having practical interest, allowing the $\rho_{2}$ dependency on $L / D_{\mathrm{PU}}$ to be neglected. With this assumption, it can be easily demonstrated that the merit function is a monotone increasing function of $\mathrm{L} / \mathrm{D}_{\mathrm{PU}}$, which optimal value is thus the maximum allowable: $\mathrm{L}_{\mathrm{PU}} \mathrm{D}^{\mathrm{Opt}}=\mathrm{L}_{\mathrm{T}} / \mathrm{D}$.

In synthesis, given a vehicle's aerodynamic shape, the optimal trajectory is obtained flying at the maximum vertical lift-to-drag ratio, and at zero bank up to the heat flux trim acquisition point. Heat flux trim is guaranteed by appropriate selection of the surface to mass ratio, i.e. such to comply with Eqs. (13), (14). Then, the bank angle has to be instantaneously changed and eventually modulated in order to exert the $\mathrm{L} / \mathrm{D}_{\mathrm{TPS}}$ required to track the constant$\dot{q}$ trajectory segment, up to the maximum allowed density, $\rho_{2}$.

\section{Approximate Model Validation}

The proposed model's applicability and effectiveness is verified by comparison with the trajectories obtained employing a standard numerical optimization technique. Being the present method a mission design tool, we expect that the approximate model preserves the main features of the optimal trajectories, but introduces some acceptable degree of approximation with respect to numeric solutions.

The numeric trajectories we consider for the comparative analysis are derived by solving a local optimization problem using a standard direct collocation scheme ${ }^{23}$. The vehicle's motion is obtained as the numeric solution of a conventional point mass 3-DOF translational model of an atmospheric entry on a spherical planet, under angle of attack and bank angle guidance ${ }^{24}$. For comparison purposes, results are shown only in terms of longitudinal variables. The US76 Standard Atmosphere model is employed to compute relevant variables, and table data are used to determine the aerodynamic forces as a function of Mach number and angle of attack.

The numeric optimization problem searches for the constant angle of attack value, the vehicle's mass, and the piecewise linear bank angle time profile that maximize the time interval in which the thermal flux is equal to the TPS test one within a given tolerance. Path constraints are enforced in terms of the maximum stagnation point heat flux, limited to be lower than $\dot{q}_{T P S}$, the maximum dynamic pressure, and a maximum bank angle rate, to model the effects of non instantaneous rotational dynamics ${ }^{25}$. The initial state is taken at the trajectory apogee, and the final 
state occurs when the flight path becomes horizontal. The latter choice allows to include in the numeric solution also the dynamic pressure constraint compliance maneuver. Indeed, imitation of the maximum dynamic pressure causes a pull-up maneuver afterwards the constant heat flux phase, that results in a subsequent $\gamma$ increase up to positive values.

The comparison is carried out considering three test cases, selected in order to be representative of the missions the method has been designed for, namely low-energy suborbital TPS flight tests. Their main characteristics are collected in Table 1, thought to cover a wide range of possible applications, at least in terms of maximum thermal flux, dynamic pressure, and initial state at the apogee. Case number 1 is derived from the mini-SRT project ${ }^{10}$, a feasibility study for a small-scale winged vehicle performed by the authors under funding by CIRA. Cases 2 and 3 refer to the same vehicle, SHARP-L1 ${ }^{11,26}$, a waverider configuration originally conceived as a follow-on of the SHARP B1 and B2 ballistic vehicles. These two cases differ in the initial states, which are taken from the X-33 suborbital trajectories studies available in open literature ${ }^{16}$. The two points of the X-33 trajectories have been used only decause of their suborbital mechanical energy content, despite the fact that the trajectories we obtain in this paper bear no similarity with the X-33 ones, that were designed to meet completely different requirements subject to dissimilar constraints. In particular, X-33 entry trajectories to Michael Army Air Field and Malmstrom Air Force Base have been used for the intial states of cases 2 and 3, respectively.

Table 1 Test cases main features

\begin{tabular}{ccccccc}
\hline \hline Test Cases & $\mathrm{L}_{\mathrm{T}} / \mathrm{D}$ & $\mathrm{R}_{\mathrm{N}}, \mathrm{cm}$ & $\dot{q}_{T P S}, \mathrm{~kW} / \mathrm{m}^{2}$ & $\bar{q}_{M a x}, \mathrm{kPa}$ & $\mathrm{E}, \mathrm{MJ} / \mathrm{kg}$ & $\mathrm{h}_{\mathrm{A}}, \mathrm{km}$ \\
\hline $\begin{array}{c}\# 1-\text { MiniSRT } \\
\# 2-\text { SHARP-L1, }\end{array}$ & 2.4 & 1.0 & 500 & 130.0 & 3.75 & 120.0 \\
$\begin{array}{c}\text { Michael Trajectory } \\
\text { \#3 - SHARP-L1, } \\
\text { Malmstrom Trajectory }\end{array}$ & 3.3 & 0.6 & 600 & 50.0 & 4.37 & 58.0 \\
\hline \hline
\end{tabular}

Results demonstrate the approximate method preserves the key features of the trajectories obtained by the standard numeric solution technique. Indeed, the analytic L/D, V and $\gamma$ profiles versus $\rho$, shown in Figs. 2 - 4, match the numeric ones within an accuracy typical of a mission design phase. In particular, it can be seen that the pull-up vertical lift-to-drag ratio derived with the approximate method is in excellent agreement with the numeric one. As expected, in the numeric trajectory the vertical lift is reduced prior than in the analytic one, because of the effect of non instantaneous rotational dynamics. In the successive TPS test phase the analytic solution envelops the numeric 
one, even though discrepancies can be observed in the TPS test end density. We remark that the approximate trajectory is shown up to this density value, computed via Eq.(23). The numeric solution instead includes also the dynamic pressure constraint compliance maneuver, represented in Fig.2 by a sudden increase in L/D.

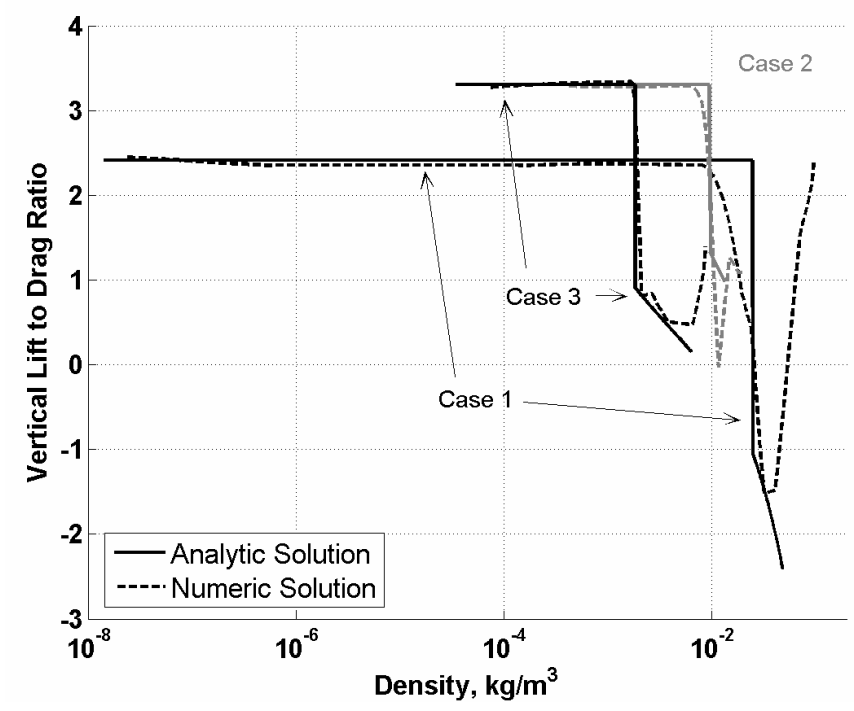

Fig. 2 Analytic vs. numeric vertical lift-to-drag ratio-density profiles.

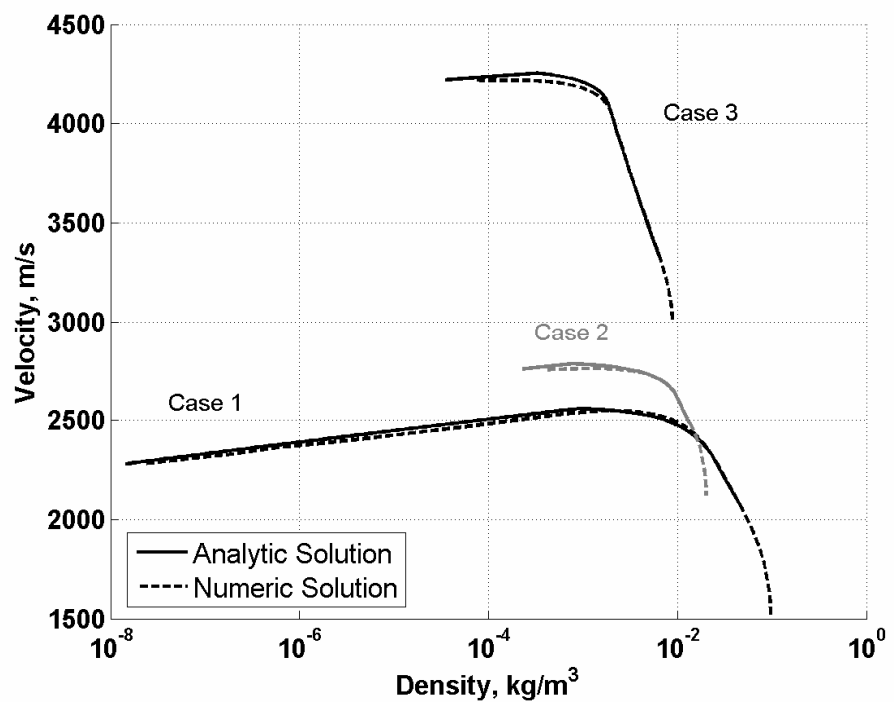

Fig. 3 Analytic vs. numeric velocity-density profiles. 


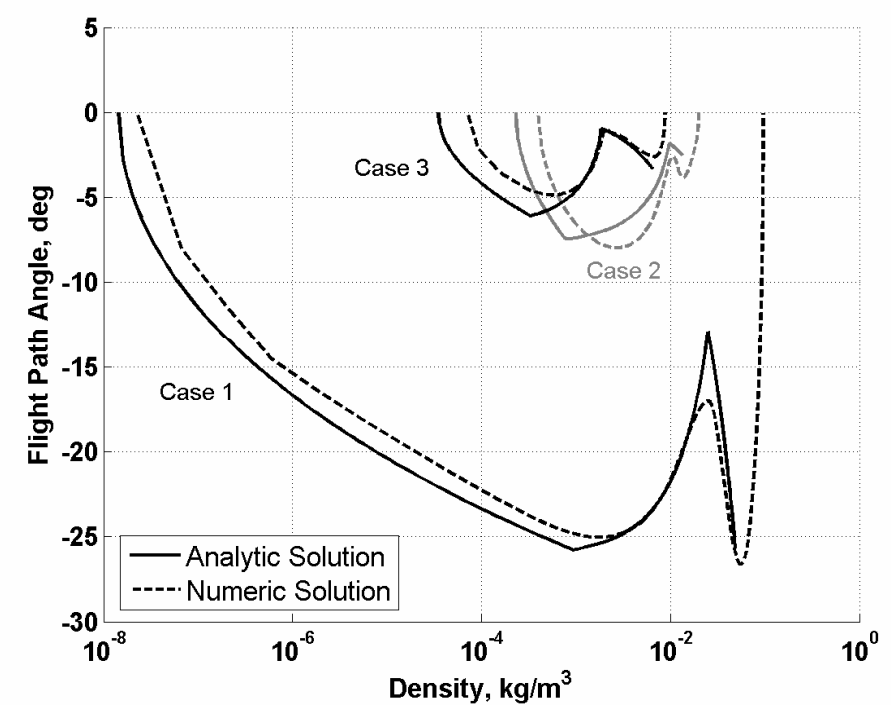

Fig. 4 Analytic vs. numeric flight path angle-density profiles.

A comparison of the resulting trajectory inputs, TPS test end density, and test time is given in Table 2. The analytic solution percentage variation w.r.t. the numeric ones is also shown. It is worth noting that the $\mathrm{B}$ and $\mathrm{L} / \mathrm{D}_{\mathrm{PU}}$ values obtained by numerical simulations vary with density. Thus, for comparison purposes, the mean values of these two inputs on the relevant density domain are used in Table 2 . Results confirm that in all the considered test cases the optimal pull-up maneuver requires maximum use of the aerodynamic efficiency in the positive vertical direction (i.e. zero bank flight), as predicted by the approximate method. The accuracy in the ballistic coefficient exhibits a certain degree of variability. Specifically, the differences between cases 1 and 3 arise from the different $\gamma$ profiles, which affect the magnitude of the error introduced by neglecting gravity tangential component. Case 2 shows a ballistic coefficient substantially higher than the other cases. In these conditions, the analytic method considerably overestimates the ballistic coefficient, because the Keplerian to atmospheric flight patching condition is expressed in terms of dynamic pressure. Indeed, the higher is B, the smaller is the drag deceleration sensed by the vehicle at a certain dynamic pressure. The resulting effect is to neglect the positive tangential gravity earlier than the other two cases, yielding a further increase in the analytically computed B. Concerning the TPS test time, modeling errors tend to balance each other yielding a more accurate $\Delta t$ estimation. For instance, overestimating B may cause either an increase or a decrease in $\Delta t$, due to the dependency on B in Eq.(7) and its effect on the $\rho_{q}$ estimate, respectively. 
Table 2 Analytic vs. numeric solution: main features comparison

\begin{tabular}{ccccccccccc}
\hline \hline & \multicolumn{3}{c}{ Case 1 } & \multicolumn{3}{c}{ Case 2 } & \multicolumn{2}{c}{ Case 3 } \\
\cline { 2 - 10 } & Analytic & Numeric & $\begin{array}{c}\% \\
\text { Variation }\end{array}$ & Analytic & Numeric & $\begin{array}{c}\% \\
\text { Variation }\end{array}$ & Analytic & Numeric & $\begin{array}{c}\% \\
\text { Variation }\end{array}$ \\
\cline { 2 - 11 }$\Delta \mathrm{t}, \mathrm{s}$ & 6.34 & 6.29 & 0.8 & 21.83 & 24.22 & -9.9 & 75.32 & 83.38 & -9.7 & -0.9 \\
$\mathrm{~L} / \mathrm{D}_{\mathrm{PU}}$ & 2.41 & 2.36 & 2.1 & 3.30 & 3.28 & 0.6 & 3.30 & 3.33 & -0.9 & -9.3 \\
$\mathrm{~B}, \mathrm{~kg} / \mathrm{m}^{2}$ & 2146.8 & 1704.1 & 26.0 & 5888.8 & 3980.3 & 47.9 & 2208.5 & 2435.2 & -9.3 \\
$\rho_{2}, \mathrm{~kg} / \mathrm{m}^{3}$ & $4.84 \mathrm{e}-2$ & $5.01 \mathrm{e}-2$ & -3.4 & $1.34 \mathrm{e}-2$ & $1.56 \mathrm{e}-2$ & -14.1 & $6.39 \mathrm{e}-3$ & $6.54 \mathrm{e}-3$ & -2.3 \\
\hline \hline
\end{tabular}

The previous analysis suggests that the semi-analytic method can adequately describe suborbital TPS flight tests trajectories for mission design purposes. Notwithstanding all the introduced approximations and the inability of analytical models to cope with angular rate limitations, the method captures the essential features of the optimal trajectories. When compared to the numeric approaches, the method proves to estimate the TPS test time and the required pull-up vertical lift-to-drag ratio with good accuracy. The accuracy in estimating the ballistic coefficient is instead variable with B itself, and it degrades as B increases.

\section{Mission Design Space Analysis}

The ultimate goal of the proposed model is to perform parametric analyses in a preliminary design phase, in order to draw meaningful indications on promising mission and system design candidates. This is done by determining the apogee conditions required to carry out a certain TPS test, given the reentry vehicle characteristics. The knowledge of the apogee conditions helps in selecting the ascent vehicle, that has a considerable effect on mission cost and complexity, and thus on weighing different design options.

In the approximate model, the TPS test and vehicle features are defined in terms of the desired heat flux and test time, the maximum admissible dynamic pressure, the vehicle's aerodynamic efficiency and the stagnation point radius. Once these parameters have been set, the apogee conditions that realize the desired mission can be obtained if the ballistic coefficient is specified. Since the ballistic coefficient is a design variable, the described approach can be used to perform parametric analyses varying B in a predetermined range.

The process yielding to the apogee conditions from the knowledge of the ballistic coefficient is described by the flow chart in Fig.5. Once a certain B is fixed, the apogee energy and altitude needed to perform the desired mission are determined by inversion of the model equations listed in Fig.5. Most of the equations can be inverted analytically. The maximum density at which the vehicle can track the heat flux trim conditions has to be determined 
solving Eq. (9) by means of a numeric root finding scheme. The Keplerian to atmospheric motion interface density needs to be determined by numeric means as well.

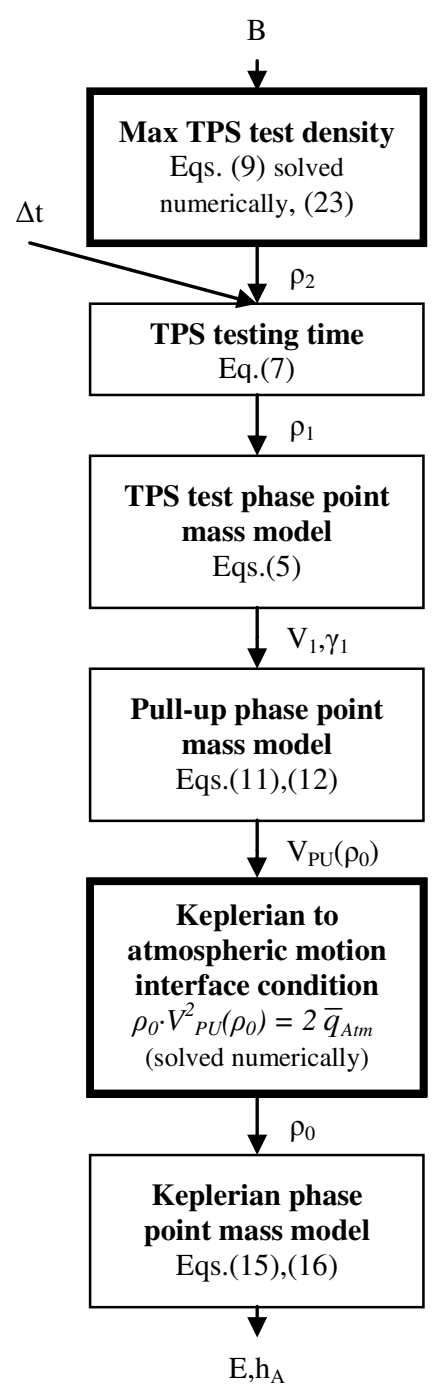

Fig. 5 Mission Design Flow Chart

In order to demonstrate the method effectiveness, a parametric analysis is carried out, using realistic values of the parameters characterizing suborbital TPS test missions (see Table 3). In all the analyses the stagnation point radius is taken equal to $1 \mathrm{~cm}$. Indeed, in order to take advantage from the higher resulting heat fluxes, sharp edges seem to be an obliged choice for suborbital TPS flight tests. While small samples may be satisfactory for materials testing, system-level testing may require flight size TPS components, e.g. to enable investigations of interface issues, and thus higher $R_{N}$ values. Although not shown here for brevity, apogee conditions required by these 
applications can be determined taking advantage of the present method. For the parametric analysis, two different test heat fluxes values are investigated, representative of the typical aero-thermal environment in lifting reentry ${ }^{9}$ and compatible with advanced TPS materials capabilities ${ }^{27}$. In addition, we will consider vehicles capable of flying at high dynamic pressures, in order to limit the apogee specific mechanical energies to suborbital values. We thus consider maximum dynamic pressure constraints ranging from $50 \mathrm{kPa}$ to $100 \mathrm{kPa}$. Two different aerodynamic efficiencies are analyzed, $\mathrm{L}_{\mathrm{T}} / \mathrm{D}=1$ and 3 , related to different aerodynamic shapes. The latter is representative of winged or lifting body configurations, whereas the former might be the efficiency of a biconic shape at nonzero angle of attack. In order for the TPS test to be of interest, a minimum test duration of 30 seconds is assumed. On the other hand, we limit the test duration to 100 seconds, since for longer times the integrated heat load could be of concern for the vehicle design. For instance, if the vehicle flies the $1 \mathrm{MW} / \mathrm{m}^{2}$ heat flux for the maximum test duration the integrated heat load would reach $100 \mathrm{MJ} / \mathrm{m}^{2}$ only in the TPS test phase, which is a non negligible fraction of typical lifting reentry heat loads ${ }^{9,11}$. Finally, the parametric analysis is conducted for ballistic coefficients ranging within $10^{3}-10^{4} \mathrm{~kg} / \mathrm{m}^{2}$, representative of physically realizable vehicles for this kind of application.

Table 3 Investigated mission design space

\begin{tabular}{cccccc}
\hline \hline $\mathrm{R}_{\mathrm{N}}, \mathrm{cm}$ & $\dot{q}_{T P S}, \mathrm{~kW} / \mathrm{m}^{2}$ & $\bar{q}_{M a x}, \mathrm{kPa}$ & $\mathrm{L}_{\mathrm{T}} / \mathrm{D}$ & $\Delta \mathrm{t}, \mathrm{s}$ & $\mathrm{B}, \mathrm{kg} / \mathrm{m}^{2}$ \\
\hline 1.0 & $\{600,1000\}$ & {$[50,100]$} & $\{1,3\}$ & {$[30,100]$} & {$\left[10^{3}, 10^{4}\right]$} \\
\hline \hline
\end{tabular}

Figure 6 shows the typical effect of $\Delta \mathrm{t}$ and $\mathrm{B}$ on apogee conditions, where the velocity is used in place of the specific mechanical energy. Indeed, expressing the results in terms of the apogee velocity allows a simpler intuitive interpretation. In addition, in suborbital flights the main contribution to the specific mechanical energy is the kinetic one, thus velocity is in practice proportional to $\sqrt{ } \mathrm{E}$. The parametric diagram shows that higher test times require higher velocities and smaller apogee altitudes. This effect becomes less significant as the ballistic coefficient increases. Results show that vehicles with high B values are preferable, being able to perform the TPS test starting from smaller specific mechanical energies. 


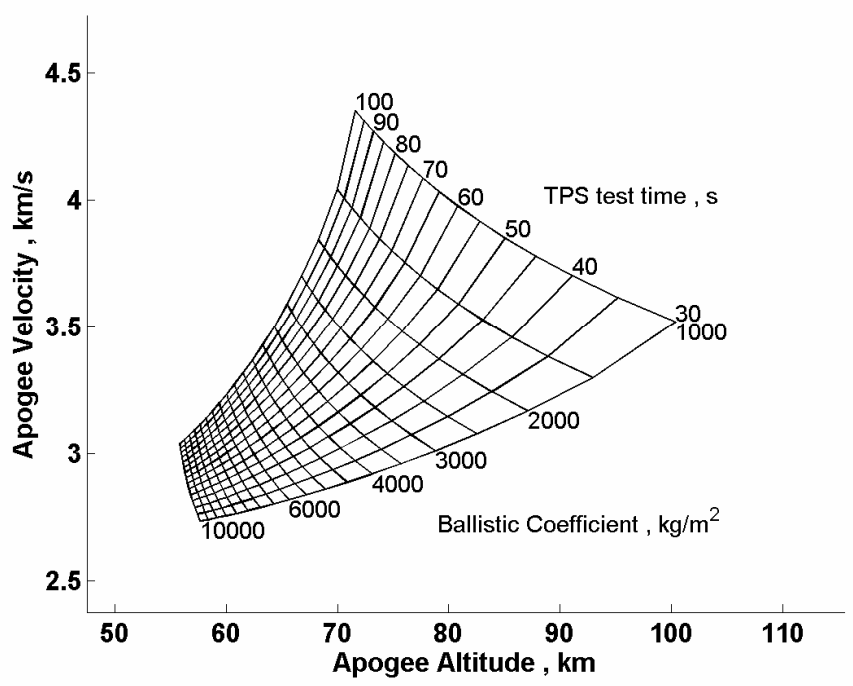

Fig. $6 \Delta t$ and B parametric effect on apogee conditions ; $\dot{q}_{T P S}=600 \mathrm{~kW} / \mathrm{m}^{2}, \mathrm{~L}_{\mathrm{T}} / \mathrm{D}=3, \bar{q}_{M a x}=100 \mathrm{kPa}$.

The effect of the maximum dynamic pressure constraint is shown in Fig.7, for a single $\dot{q}_{T P S}, \mathrm{~L}_{\mathrm{T}} / \mathrm{D}$, and $\Delta \mathrm{t}$ combination. Increasing the maximum sustainable dynamic pressure allows reducing the specific kinetic energy at the apogee, whereas the apogee altitude has to be raised. This results into a lower specific mechanical energy, especially for high B values, that are preferable for energy minimization.

Figure 8 shows the maximum trackable densities as given by the $\dot{q}_{T P S}$ tracking requirement ( Eq. (9) ) and the dynamic pressure constraint compliance maneuver ( Eq. (22) ). It is worth noting that, as anticipated in section III, the maximum TPS test density is dictated by the dynamic pressure constraint in almost all the analyzed cases. Indeed, even considering the biggest $\rho_{q}$, arising from the highest $\bar{q}_{M a x}$, the density value given by the $\dot{q}_{T P S}$ tracking requirement is almost always higher than $\rho_{\mathrm{q}}$. There are only a limited number of conditions in which this is not true, occurring for low $\mathrm{B}-$ low $\mathrm{L}_{\mathrm{T}} / \mathrm{D}$ values, thus having a limited practical interest. 


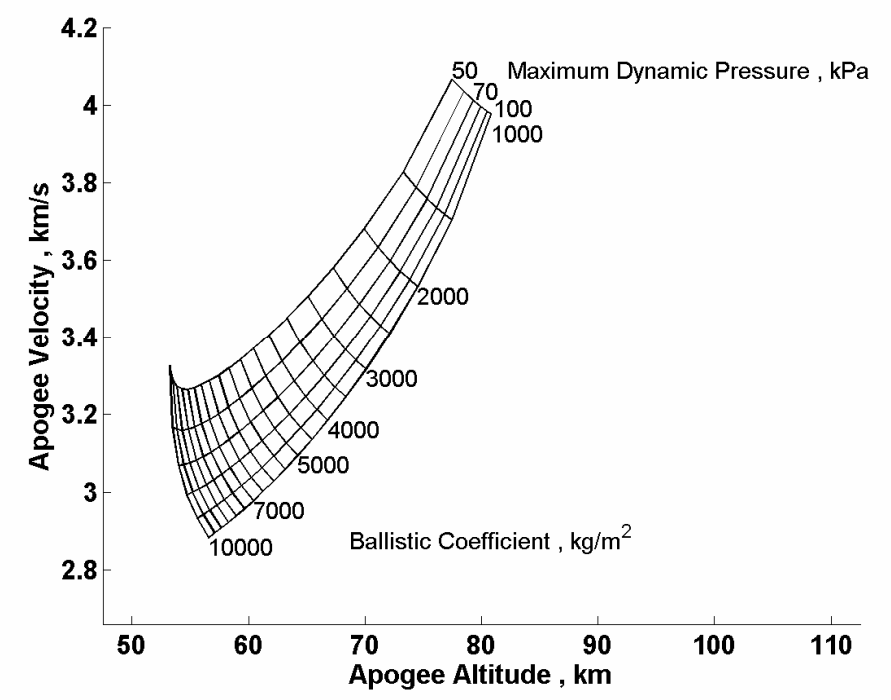

Fig. 7 Dynamic pressure constraint effect on apogee conditions ; $\dot{q}_{T P S}=600 \mathrm{~kW} / \mathrm{m}^{2}, \mathrm{~L}_{\mathrm{T}} / \mathrm{D}=3, \Delta \mathrm{t}=60 \mathrm{~s}$.
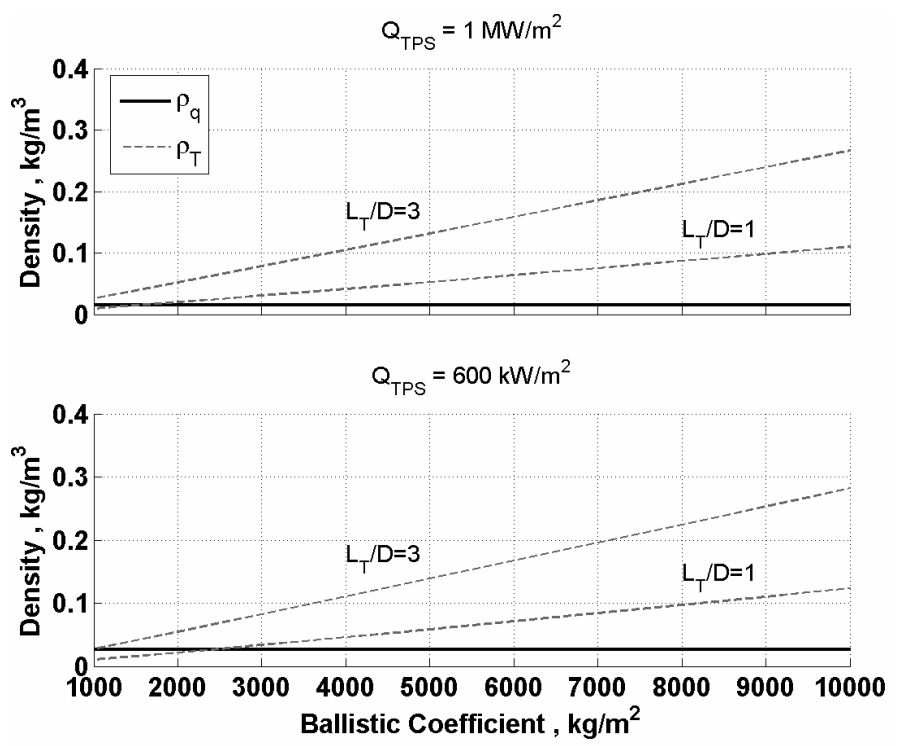

Fig. 8 Dynamic pressure constraint compliance vs. maximum trackable densities; $\bar{q}_{M a x}=100 \mathrm{kPa}$.

Different mission design options can be compared using apogee velocity and altitude parametric diagrams. As an example, Figs.9, 10 collect the diagrams of different design options for a $600 \mathrm{~kW} / \mathrm{m}^{2}$ test heat flux. The low aerodynamic efficiency, low maximum dynamic pressure case is not shown, given the unfavorable effect of this combination on the apogee conditions. In addition, low aerodynamic efficiency vehicles can be designed to withstand a high dynamic pressure flight more easily than winged ones. Results show that low $\mathrm{L}_{\mathrm{T}} / \mathrm{D}$ vehicles are not capable of reaching the required heat flux trim conditions if their ballistic coefficient is higher than $\sim 6000 \mathrm{~kg} / \mathrm{m}^{2}$. Even for lower B values the maximum achievable test duration can be shorter than 100 seconds. Interestingly, in this 
design option there is a B value that minimizes the Keplerian phase specific mechanical energy, equal to $\sim 5000$ $\mathrm{kg} / \mathrm{m}^{2}$ for test times around $30 \mathrm{~s}$. The existence of an optimal ballistic coefficient is justified by the complex dependency of initial conditions on B, that causes opposite effects. For instance, increasing B allows to start the TPS test at a lower velocity, with a consequent reduction in the required initial energy. Conversely, a higher energy is needed to perform a given pull-up with a vehicle less sensible to aerodynamic accelerations.

The comparison with the other two design options shows that the advantage of operating with higher maneuverability vehicle $\left(\mathrm{L}_{\mathrm{T}} / \mathrm{D} 1\right.$ versus 3$)$ is lost if the maximum sustainable dynamic pressure is halved. Indeed, if B is higher than $\sim 2500 \mathrm{~kg} / \mathrm{m}^{2}$ the low $\mathrm{L}_{\mathrm{T}} / \mathrm{D}$, high $\bar{q}_{\text {Max }}$ option yields lower apogee energies. Nevertheless, if the higher aerodynamic efficiency vehicle can withstand the same dynamic pressure, this option is the most favorable since the same TPS test can be performed with a smaller apogee energy. For completeness, the three design options are compared in Table 4, showing the apogee conditions required when $\mathrm{B}=5000 \mathrm{~kg} / \mathrm{m}^{2}$ and $\Delta \mathrm{t}=30 \mathrm{~s}$.

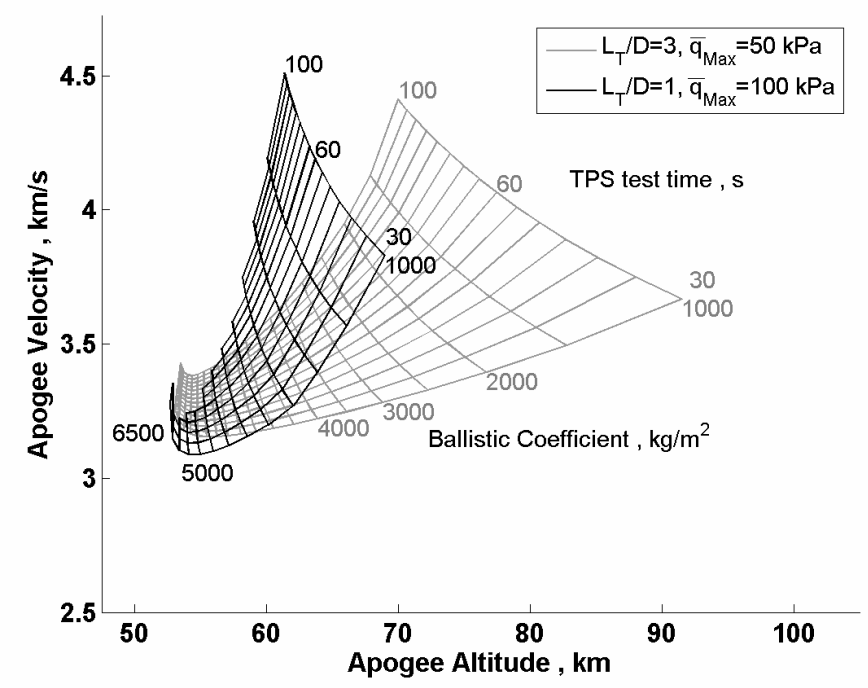

Fig. 9 Apogee conditions for different design options; $\dot{q}_{T P S}=600 \mathrm{~kW} / \mathrm{m}^{2}$. 


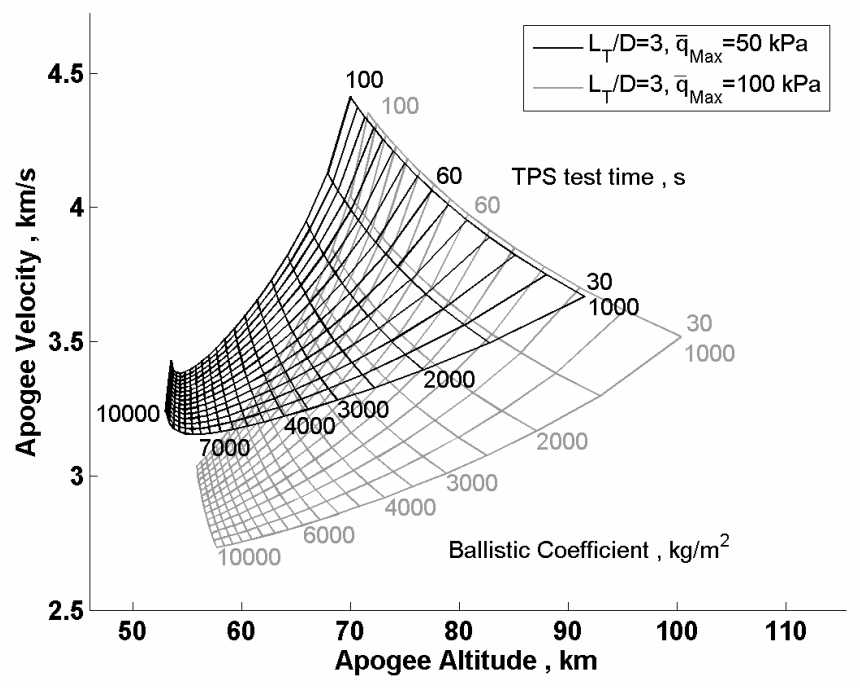

Fig. 10 Apogee conditions for different design options; $\dot{q}_{T P S}=600 \mathrm{~kW} / \mathrm{m}^{2}$.

Table 4 Example apogee conditions; $\dot{q}_{T P S}=600 \mathrm{~kW} / \mathrm{m}^{2}, \mathrm{~B}=5000 \mathrm{~kg} / \mathrm{m}^{2}$, and $\Delta \mathrm{t}=30 \mathrm{~s}$

\begin{tabular}{ccccc}
\hline \hline $\mathrm{L}_{\mathrm{T}} / \mathrm{D}$ & $\bar{q}_{\mathrm{Max}}, \mathrm{kPa}$ & $\mathrm{V}_{\mathrm{A}}, \mathrm{m} / \mathrm{s}$ & $\mathrm{h}_{\mathrm{A}}, \mathrm{km}$ & $\mathrm{E}, \mathrm{MJ} / \mathrm{kg}$ \\
\hline 1 & 100 & 3089.7 & 55.0 & 5.31 \\
3 & 100 & 2860.1 & 68.9 & 4.76 \\
3 & 50 & 3186.8 & 60.5 & 5.67 \\
\hline \hline
\end{tabular}

From the previous analysis the maximum dynamic pressure turns out to have a significant effect on the achievable performance. For this reason, results of the more demanding $1 \mathrm{MW} / \mathrm{m}^{2}$ case study, shown in Fig.11, are plotted only for vehicles capable to withstand a dynamic pressure of $100 \mathrm{kPa}$. The low maneuverability alternative is unable to pull-up to the desired heat flux trim conditions for ballistic coefficients higher than $2500 \mathrm{~kg} / \mathrm{m}^{2}$. Moreover, the high $\mathrm{L}_{\mathrm{T}} / \mathrm{D}$ vehicle exhibits an optimal B value around $6000-6500 \mathrm{~kg} / \mathrm{m}^{2}$, that slightly depends on the desired test duration. Since this vehicle option also has the highest performances among the analyzed ones, we can conclude that for TPS testing at $\dot{q}_{T P S}=1 \mathrm{MW} / \mathrm{m}^{2}$ there are optimal vehicle designs in the investigated parameters ranges, that minimize the specific mechanical energy at the trajectory apogee. Their main features are collected in Table 5 for minimum and maximum TPS test durations. 


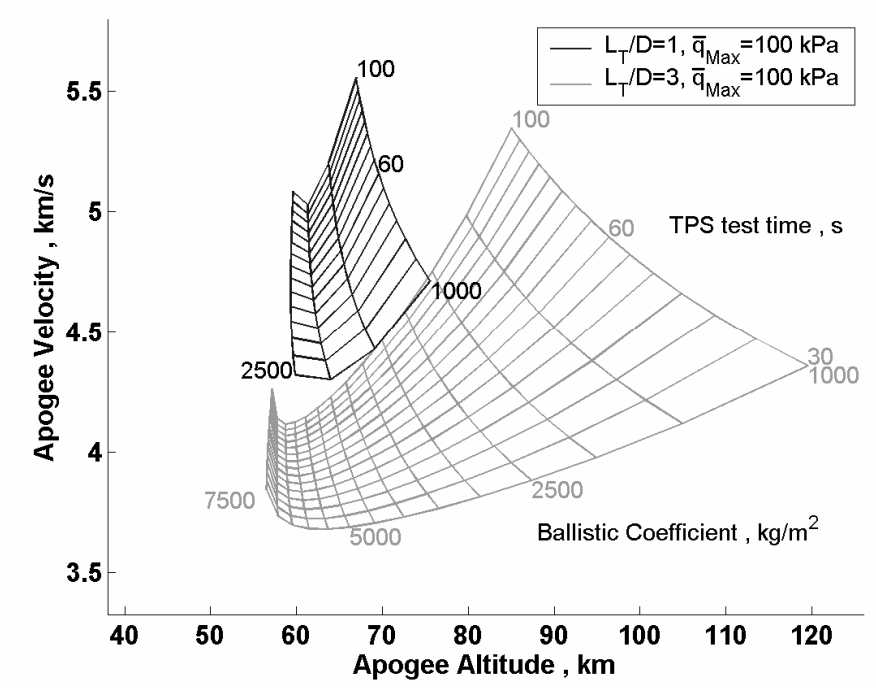

Fig. 11 Apogee conditions for different design options; $\dot{q}_{T P S}=1000 \mathrm{~kW} / \mathrm{m}^{2}$.

Table 5 Optimal mission designs; $\dot{q}_{T P S}=1 \mathrm{MW} / \mathrm{m}^{2}, \bar{q}_{M a x}=100 \mathrm{kPa}, \mathrm{L}_{\mathrm{T}} / \mathrm{D}=3$

\begin{tabular}{ccccc}
\hline$\Delta \mathrm{t}, \mathrm{s}$ & $\mathrm{B}, \mathrm{kg} / \mathrm{m}^{2}$ & $\mathrm{~V}_{\mathrm{A}}, \mathrm{m} / \mathrm{s}$ & $\mathrm{h}_{\mathrm{A}}, \mathrm{km}$ & $\mathrm{E}, \mathrm{MJ} / \mathrm{kg}$ \\
\hline 30 & 5934 & 3681.3 & 61.7 & 7.38 \\
100 & 6467 & 4116.5 & 58.8 & 9.04 \\
\hline \hline
\end{tabular}

\section{Conclusion}

An approximate methodology has been developed and validated, that identifies an optimal mission profile for an advanced TPS suborbital flight test, designed to expose a specific area of the vehicle to a constant heat flux. The methodology requires the numeric solution of two scalar nonlinear equations and the a priori knowledge only of high level mission requirements, such as the desired TPS test heat flux. These features make it appealing to perform parametric analyses in broad regions of the mission design space, in order to limit promising design candidates to an extent manageable by conventional numeric-simulation-based methods. The approximate method is capable of identifying with a minimum effort the trajectory apogee conditions required to perform the test as desired. This information is of great usefulness in selecting the needed launch vehicle. Indeed, the apogee conditions achievable by a generic rocket can be evaluated in a relatively straightforward manner, and then compared to those computed by the approximate method.

In order to demonstrate its effectiveness, a mission analysis has been performed using reasonable ranges of relevant parameters. Results show that needed apogee conditions considerably differ from the ones provided by existing off-the-shelf vectors. For instance, sounding rockets, that seem the most convenient choice for cost 
constriction, could attain the needed specific mechanical energies, but are designed for considerably higher apogee altitudes. Their possible adaptation to the required apogee conditions would call for major changes, even at a system level. Therefore, even if the ascent vehicle selection is beyond the scope of this work, the use of the proposed method suggests that a consistent effort would be required to obtain the constant heat flux TPS test apogee conditions with off-the-shelf rockets. Nevertheless, the constant heat flux flight test can be weighed against other TPS testing alternatives taking advantage of the proposed tool.

In terms of the entry phase mission design, results point out that increasing the vehicle's ballistic coefficient allows reducing the specific mechanical energy at reentry, even though there is a superior limit above which this behavior is reversed. The maximum admissible dynamic pressure turns out to have a primary effect on the TPS testing capabilities of a candidate design. For instance, cases are shown in which a biconic shaped vehicle can accomplish the same mission of a winged one with smaller launch energies, since it is capable of withstanding a higher dynamic pressure.

Finally, the proposed tool is capable to identify the existence of optimum solutions in the mission desing space. Indeed, concerning test heat fluxes around $1 \mathrm{MW} / \mathrm{m}^{2}$, representative of limit capabilities of novel TPS ceramic materials, an optimal mission design is found in the investigated parameters region. Specifically, results show that using the optimal design to reproduce this severe aero-thermal environment for 100 seconds requires an apogee specific mechanical energy 30 percent lower than the circular orbit one at the same altitude.

\section{References}

${ }^{1}$ Vennemann, D., "Hypersonic test facilities available in Western Europe for aerodynamic/aerothermal and structure/material investigations," Philosophical Transactions: Mathematical, Physical and Engineering Sciences, Vol. 357, No. 1759, Aug. 1999, pp. $2227-2248$.

${ }^{2}$ Stewart, D., and Venkatapathy, E., "Thermal Protection System Evaluation Using Arc-jet Flows: Flight Simulation or Research Tool?", 105th Annual Meeting and Exposition of the American Ceramic Society, Nashville, TN, United States, Apr. 2003.

${ }^{3}$ Yoshinaga, T., Tate, A., Watanabe, M., and Shimoda, T., "Orbital re-entry experiment vehicle ground and flight dynamic test results comparison," AIAA Journal of Spacecraft and Rockets, Vol. 33, No. 5, 1996, pp. 635-642. 
${ }^{4}$ Muylaert, J., Cipollini, F., Tumino, G., Kordulla, W., Saccoccia, G., Stavrinidis, C., Caporicci, M., Walpot, L., Ottens, H., ”Preparing for Atmospheric Reentry With EXPERT's Help - An Aerothermodynamic In-Flight Research Programme,” ESA Bulletin, No. 114 , May 2003, pp. 42-48, URL: http://www.esa.int/esapub/bulletin/bullet114/chapter4_bul114.pdf

${ }^{5}$ Anon., "SOAREX (Sub-Orbital Aerodynamic Re-entry EXperiments),” NASA Ames technology Capabilities and Facilities, [online], URL: http://www.nasa.gov/centers/ames/research/technology-onepagers/soarex.html

${ }^{6}$ Eggers, T., Longo, J., Hoerschgen, M. and Stamminger, A., “The Hypersonic Flight Experiment SHEFEX ," AIAA/CIRA 13th International Space Planes and Hypersonics Systems and Technologies Conference, Capua, Italy, May 16-20, 2005.

${ }^{7}$ Rasky, D., Salute, J., Kolodziej, P., Bull, J., “The NASA Sharp Flight Experiment,” 3rd European Workshop on Thermal Protection Systems, Noordwijk, Netherlands, 25-27 Mar. 1998.

${ }^{8}$ Russo, G., "USV flying test beds for future generations LV technology development," 12th AIAA International Space Planes and Hypersonic Systems and Technologies, AIAA 2003-6978, Norfolk, VA, December 2003.

${ }^{9}$ Ried, R.C., "Orbiter Entry Aerothermodynamics," Space Shuttle Technical Conference, NASA CP-2342, Part 2, Houston, TX, 1983, pp. 1051-1061.

${ }^{10}$ Tancredi, U., Accardo, D., Grassi M., and Curreri, F., "Unmanned space vehicle technology demonstrator," Acta Astronautica, Vol. 60, No. 3, 2007, pp. 186-197.

${ }^{11}$ Tillier, C. E., "Simulation-Based Analysis of Reentry Dynamics for the Sharp Atmospheric Entry Vehicle," NASA CR1998-208334, 1998.

${ }^{12}$ Betts, J.T., "Survey of Numerical Methods for Trajectory Optimization," AIAA Journal of Guidance, Control, and Dynamics, Vol. 21, No. 2, 1998, pp. 193-207.

${ }^{13}$ Murray, J. E., and Tartabini, P. V., “Development of a Mars Airplane Entry, Descent, and Flight Trajectory”, NASA TM2001-209035, 2001.

${ }^{14}$ Griffin, M.D., French, J.R., Space Vehicle Design, AIAA Education Series, AIAA, Washington DC, 1991, Chaps. 5, 6.

${ }^{15}$ Harpold, J.D. and Graves, C.A., Jr., "Shuttle Entry Guidance”, Journal of the Astronautical Sciences, Vol. 27, No. 3, 1979, pp. 239-268.

${ }^{16}$ Lu, P., Hanson, J.M., "Entry Guidance for the X-33 Vehicle", AIAA Journal of Spacecraft and Rockets, Vol. 35, No. 3, 1998, pp. 342-349.

${ }^{17}$ Zimmerman, C., Dukeman, G., Hanson, J., “Automated Method to Compute Orbital Reentry Trajectories with Heating Constraints”, AIAA Journal of Guidance, Control, and Dynamics, Vol.26, No.4, 2003, pp. 523-529.

${ }^{18}$ Savino, R. , De Stefano Fumo, M., Paterna, D., Serpico, M., “Aerothermodynamic study of UHTC-based thermal protection systems”, Aerospace Science and Technology, Vol.9, No. 2, 2005, pp. 151-160. 
${ }^{19}$ Kuntz, D., Potter, D., "Boundary Layer Transition and Hypersonic Flight Testing," 45th AIAA Aerospace Sciences Meeting and Exhibit, AIAA-2007-308, Reno, Nevada, January 2007.

${ }^{20}$ Fay, J.A., and Riddell, F.R., "Theory of stagnation point heat transfer in dissociated air," Journal of Aeronautical Sciences, Vol 25, February 1958, pp. 73-85.

${ }^{21}$ Loh, W.H.T, Re-entry and Planetary Entry Physics and Technology, Springer-Verlag, New York, 1968.

${ }^{22}$ Regan, F. J., Anandakrishnan, S. M., Dynamics of Atmospheric Re-Entry, AIAA Education Series, AIAA, Washington DC, 1993, Chaps. 6, 7.

${ }^{23}$ Hull, D.G. "Conversion of Optimal Control Problems into Parameter Optimization Problems", AIAA Journal of Guidance, Control, and Dynamics, Vol.20, No.1, 1997, pp. 57-60.

${ }^{24}$ Vinh, N. X., Busemann, A., and Culp, R. D., Hypersonic and Planetary Entry Flight Mechanics, The University of Michigan Press, Ann Arbor, MI, 1980, pp. 26-27.

${ }^{25}$ Jits, R.Y., Walberg, G.D., "Blended control, predictor-corrector guidance algorithm: an enabling technology for Mars aerocapture", Acta Astronautica, Vol. 54, No. 6, 2004, pp. 385 - 398.

${ }^{26}$ Kolodziej, P. , Bowles, J. V., Brown, J. L., Cornelison, C. J., Lawrence, S. L., Loomis, M. P., Merriam, M. L., Rasky, D. J., Tam, T. C., Wercinski P. F., "Sharp-L1 technology demonstrator development - An aerothermodynamic perspective," 34th AIAA Thermophysics Conference, AIAA-2000-2688, Denver, CO, 2000.

${ }^{27}$ Ogasawara, K., Nagase, T., Yamao, H., and Fujii, K., "Reentry Vehicle Crossrange Ability Improvement by Using High Temperature Ceramics," 11th AIAA/AAAF International Conference, Space planes and Hypersonic Systems and Technologies, Orlèans, France, October 2002. 